\title{
Carbohydrate antigen 19-9 as a prognostic biomarker in pancreatic neuroendocrine tumors
}

\author{
GUOPEI LUO ${ }^{1-3^{*}}$, KAIZHOU JIN ${ }^{1-3^{*}}$, HE CHENG $^{1-3^{*}}$, CHEN LIU $^{1-3}$, MENG GUO $^{1-3}$,

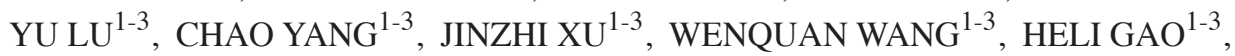 \\ SHIRONG ZHANG ${ }^{1-3}$, JIANG LONG ${ }^{1-3}$, JIN XU ${ }^{1-3}$, QUANXING NI ${ }^{1-3}$, JIE CHEN $^{4}$ and XIANJUN YU ${ }^{1-3}$ \\ ${ }^{1}$ Department of Pancreatic Surgery, Fudan University Shanghai Cancer Center, Shanghai; \\ ${ }^{2}$ Department of Oncology, Shanghai Medical College, Fudan University; ${ }^{3}$ Pancreatic Cancer Institute, \\ Fudan University, Shanghai 200032; ${ }^{4}$ Department of Gastroenterology, \\ The First Affiliated Hospital, Sun Yat-Sen University, Guangzhou, Guangdong 510080, P.R. China
}

Received December 20, 2016; Accepted March 7, 2017

DOI: $10.3892 / \mathrm{ol} .2017 .7071$

\begin{abstract}
Carbohydrate antigen 19-9 (CA19-9) is not generally considered to be a biomarker in pancreatic neuroendocrine tumors (pNETs), as the majority of pNETs present with a normal range of CA19-9. The present study aimed to evaluate the role of serum CA19-9 levels as a prognostic factor in a relatively large number of patients with pNETs. Consecutive patients were retrospectively collected from a single institution between June 2006 and February 2015. The receiver operating characteristic (ROC) curve and the area under the ROC curve were used to select the cut-off values for the baseline CA19-9 levels. The primary end point was set as overall survival. Potential factors associated with the abnormal elevation of CA19-9 expression levels in pNETs were also investigated. The cut-off value for CA19-9 was $16 \mathrm{U} / \mathrm{ml}$ as determined by the ROC curve, and for the area under the ROC curve it was 0.68. In total, $32.7 \%$ of patients (51/156) had CA19-9 expression levels higher than the cut-off value. Univariate analysis demonstrated that CA19-9 >16 U/ml was an adverse prognostic factor for patients' overall survival. The CA19-9 >16 U/ml group had a statistically higher proportion of tumor node metastasis (TNM) stage III or IV, as compared with the CA19-9 $\leq 16 \mathrm{U} / \mathrm{ml}$ group. To the best of our knowledge, the present study is the
\end{abstract}

Correspondence to: Dr Xianjun Yu, Department of Pancreatic Surgery, Fudan University Shanghai Cancer Center, 270, Dong'An Road, Xuhui, Shanghai 200032, P.R. China

E-mail: yuxianjun@fudanpci.org

Dr Jie Chen, Department of Gastroenterology, The First Affiliated Hospital, Sun Yat-Sen University, 58 Zhongshan II Road, Guangzhou, Guangdong 510080, P.R. China

E-mail: chen0jie@hotmail.com

*Contributed equally

Key words: pancreatic neuroendocrine tumors, prognosis, carbohydrate antigen 19-9, biomarker first to demonstrate that CA19-9 is a prognostic biomarker of pNETs, one that may reflect its aggressiveness and severity.

\section{Introduction}

Pancreatic neuroendocrine tumors (pNETs) are tumors arising from the endocrine cells of the pancreas; pNETs comprise $<3 \%$ of novel pancreatic neoplasms (1). Its incidence has increased in recent years since the introduction of novel diagnostic procedures (2). pNETs can be generally divided into functional and nonfunctional tumors. Nonfunctional pNETs constitute $\sim 85 \%$ of all pNETs and are more aggressive compared with the functional pNETs $(1,3)$. Although they are generally viewed as indolent tumors, pNETs are highly heterogenous neoplasms and certain subgroups may demonstrate aggressive characteristics $(4,5)$. Currently, therapeutic methods for pNETs are diverse, including surgical resection and non-surgical interventions (targeted therapies, chemotherapy, somatostatin analogues, peptide receptor radionuclide therapy and liver-directed therapies) (6-8). Close observation may be required to small pNETs $(9,10)$. Therefore, biomarkers that reflect the aggressive features of pNETs are urgently required in order to aid therapeutic decisions and follow-up observations (11).

Carbohydrate antigen 19-9 (CA19-9) is a tumor-associated carbohydrate biomarker that was derived from a human colorectal cancer cell line targeted by the monoclonal antibody 1116-NS-19-9 (12). It has been widely used in the management of gastrointestinal malignancies, particularly for pancreatic cancer $(11,13)$. In a pool data analysis of CA19-9 for the diagnosis of pancreatic cancer, the median sensitivity was $79 \%$ (70-90\%) and the median specificity was $82 \%$ (68-91\%) (14). It is a sialylated Lewis blood group antigen and its secretion is influenced by Lewis antigen phenotypes (13). The elevation of CA19-9 expression levels has also been observed in other conditions, including biliary obstruction and inflammation, digestive tract inflammation and other gastrointestinal malignancies, which limits its clinical application in pancreatic cancer $(13,14)$.

CA19-9 is not generally considered to be a diagnostic or prognostic biomarker in pNETs as the majority of pNETs 
present with normal range of CA19-9 $(15,16)$. Conversely, in view of its abnormal increased expression level in common pancreatic cancer; CA19-9 has been used as a diagnostic marker to differentiate pancreatic cancer from pNETs $(15,16)$. However, few previous studies have demonstrated that CA19-9 may be used as a prognostic biomarker in neuroendocrine tumors $(17,18)$. For example, Elisei et al $(17)$ reported a case of multiple endocrine neoplasia type $2 \mathrm{~B}$ with significant elevation of CA19-9 expression levels. The patient experienced rapid disease progression and survived for only a short period, indicating that CA19-9 may be a biomarker of aggressiveness (17). A further study conducted by Elisei et al (18) revealed that 16/100 advanced structural recurrent/persistent medullary thyroid cancer tissues exhibited high expression levels of CA19-9, and the CA19-9 positive group demonstrated a higher mortality rate compared with the normal CA19-9 expression group.

The aim of current study was to evaluate the role of serum CA19-9 expression levels as a prognostic factor in a relatively large cohort of patients with pNETs at various clinical stages. Potential factors associated with abnormally increased expression levels of CA19-9 in pNETs were also investigated.

\section{Materials and methods}

Study design and treatment. Patients (156 cases) were retrospectively retrieved from a single institution (Shanghai Cancer Center, Fudan University, Shanghai, China) between June 2006 and February 2015. The male-female ratio was $\sim 1.1: 1$, with a mean age of 53 (range, 15-77). Specimens were collected prior to initiating major treatment by the Tissue Bank, Shanghai Cancer Center (Shanghai, China). The enrollment criteria included subjects who had baseline CA19-9 information. In addition, patients were included if they had complete demographics information. Patients were excluded if the diagnosis of pNET was not pathologically confirmed. All the cases were staged according to the modified European Neuroendocrine Tumor Society Tumor-Node-Metastasis (TNM) staging system (19). Tumors were classified as G1, G2 or G3 according to the 2010 World Health Organization classification (based on the mitotic index and the Ki-67 index) (20). All clinical and pathological data were collected from patient medical records obtained from the Shanghai Cancer Center, Fudan University. Incidental pNETs were detected during health check-ups or evaluations for unassociated symptoms (15). Functional pNETs were also included in incidental pNETs in the present study. The primary end point was set as overall survival. Follow-up information was updated in December 2016, with a median follow-up time of 32 months. Survival time was determined from the date of final diagnosis to the date of the last follow-up or mortality. The present study was approved by the Ethical Committee of Shanghai Cancer Center, Fudan University. Informed written consent was obtained from all patients prior to enrollment in the current study.

CA19-9 evaluation. Serum CA19-9 expression levels were examined within 2 weeks prior to major treatment initiation using an electrochemiluminescence immunoassay on the Roche Cobas e601 (Roche MODU D+P model, D2400-P800) immunoassay analyzer (Roche Diagnostics $\mathrm{GmbH}$,
Table I. Demographics and clinical characteristics.

Demographic/clinical characteristics

Patients, n (\%)

\begin{tabular}{|c|c|}
\hline \multicolumn{2}{|l|}{ Age } \\
\hline$\leq 50$ years & $69(44.2)$ \\
\hline$>50$ years & $87(55.8)$ \\
\hline \multicolumn{2}{|l|}{ Gender } \\
\hline Male & $73(46.8)$ \\
\hline Female & $83(53.2)$ \\
\hline \multicolumn{2}{|l|}{ Location } \\
\hline Head & 65 (41.7) \\
\hline Other & $91(58.3)$ \\
\hline \multicolumn{2}{|l|}{ Size } \\
\hline$\leq 3 \mathrm{~cm}$ & $53(34.0)$ \\
\hline$>3 \mathrm{~cm}$ & $103(66.0)$ \\
\hline \multicolumn{2}{|l|}{ TNM stage } \\
\hline I, II & $102(65.4)$ \\
\hline III, IV & $54(34.6)$ \\
\hline \multicolumn{2}{|l|}{ Grade } \\
\hline $\mathrm{G} 1, \mathrm{G} 2$ & $114(73.1)$ \\
\hline G3 & $16(10.3)$ \\
\hline Unknown & $26(16.7)$ \\
\hline \multicolumn{2}{|l|}{ Functional } \\
\hline Positive & $15(9.6)$ \\
\hline Negative & $141(90.4)$ \\
\hline \multicolumn{2}{|c|}{ Symptomatic } \\
\hline Positive & $92(59.0)$ \\
\hline Negative & $64(41.0)$ \\
\hline
\end{tabular}

TNM, tumor-node-metastasis.

Mannheim, Germany) according to the manufacturer's protocol. The recommended upper limit for the serum CA19-9 expression level was $<37 \mathrm{U} / \mathrm{ml}$. CA19-9 has been reported to be affected by biliary obstruction, inflammation and other conditions (14); thus, all patients with serum bilirubin $>2 \mathrm{mg} / \mathrm{ml}$, biliary obstruction and inflammation, digestive tract inflammation or other gastrointestinal malignancies at the time of CA19-9 evaluation were excluded (165 cases were included at the start and 156 cases were included subsequent to the exclusions).

Statistical analysis. The receiver operating characteristic (ROC) curve and area under the ROC curve were used to select the optimal cut-off values for baseline CA19-9 expression levels. Time-to-event variables were determined using the Kaplan-Meier method. Arms stratified by potential prognostic factors (age, gender, size, location, TNM stage, CA19-9 levels, grade and symptom) were analyzed by the log-rank tests. The Cox's proportional hazard ratio (HR) with a $95 \%$ confidence interval (CI) was used to estimate the difference between the stratified arms using the Stata ${ }^{\circledR}$ version 12.0 statistical software package (StataCorp LP, College Station, TX, USA). Categorical data were analyzed 
Table II. Univariate and multivariate analysis for overall survival of all patients using the Cox proportional hazards model.

\begin{tabular}{|c|c|c|c|c|c|c|}
\hline \multirow[b]{2}{*}{ Characteristic } & \multicolumn{3}{|c|}{ Univariate analysis } & \multicolumn{3}{|c|}{ Multivariate analysis } \\
\hline & HR & $95 \%$ CI & P-value & HR & $95 \%$ CI & P-value \\
\hline \multicolumn{7}{|l|}{ Age } \\
\hline$\leq 50$ & 1 & l & l & & & \\
\hline$>50$ & 0.82 & $0.40-1.67$ & 0.581 & & & \\
\hline \multicolumn{7}{|l|}{ Gender } \\
\hline Male & 1 & l & 1 & & & \\
\hline Female & 0.52 & $0.25-1.08$ & 0.075 & & & \\
\hline \multicolumn{7}{|l|}{ Size $(\mathrm{cm})$} \\
\hline$\leq 3$ & 1 & l & 1 & & & \\
\hline$>3$ & 1.88 & $0.81-4.38$ & 0.135 & & & \\
\hline \multicolumn{7}{|l|}{ Location } \\
\hline Head & 1 & l & 1 & & & \\
\hline Other & 1.16 & $0.56-2.41$ & 0.682 & & & \\
\hline \multicolumn{7}{|l|}{ TNM stage } \\
\hline I, II & 1 & l & 1 & 1 & l & l \\
\hline III, IV & 5.08 & $2.42-10.67$ & $<0.001$ & 2.88 & $1.20-6.93$ & $0.018^{\mathrm{a}}$ \\
\hline \multicolumn{7}{|l|}{ CA19-9 (U/ml) } \\
\hline$\leq 16$ & 1 & l & l & 1 & 1 & l \\
\hline$>16$ & 2.60 & $1.28-5.28$ & 0.006 & 1.52 & $0.70-3.29$ & 0.286 \\
\hline \multicolumn{7}{|l|}{ Grade } \\
\hline $\mathrm{G} 1, \mathrm{G} 2$ & 1 & l & l & 1 & 1 & l \\
\hline G3 & 13.43 & $5.65-31.93$ & $<0.001$ & 8.79 & $3.47-22.28$ & $<0.001^{\mathrm{a}}$ \\
\hline Unknown & 3.10 & $1.25-7.69$ & 0.015 & 1.77 & $0.65-4.80$ & 0.262 \\
\hline \multicolumn{7}{|l|}{ Incidental } \\
\hline Negative & 1 & l & l & & & \\
\hline Positive & 0.67 & $0.30-1.45$ & 0.295 & & & \\
\hline
\end{tabular}

${ }^{\mathrm{a}} \mathrm{P}<0.05$; TNM, tumor-node-metastasis; HR, hazard ratio; CI, confidence interval.

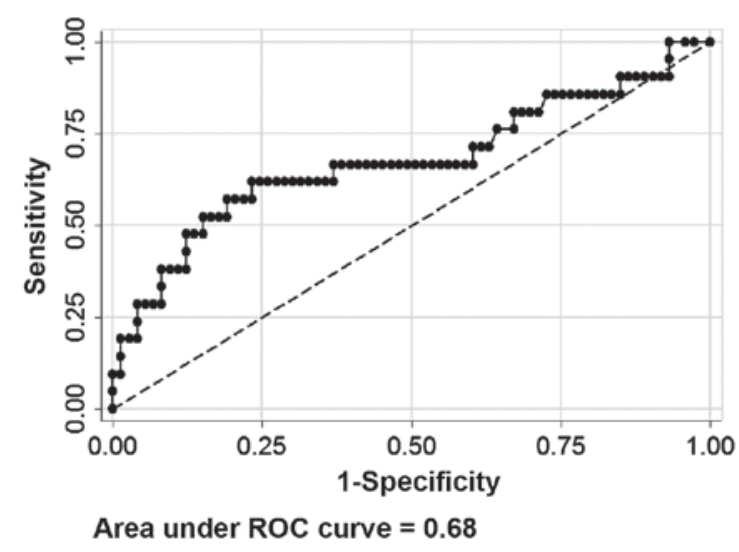

Figure 1. The ROC curve and area under the ROC curve for baseline carbohydrate antigen 19-9 expression levels as a prognostic factor. ROC, receiver-operating characteristic.

using Pearson's $\chi^{2}$ test or Fisher's exact test as appropriate. A two-sided $\mathrm{P}<0.05$ was considered to indicate a statistically significant difference.

\section{Results}

Data and survival analysis of patients. A total of 156 patients with pathologically confirmed pNETs were included in the final analysis (Table I). The male-female ratio was $\sim 1.1: 1$, with $55.8 \%$ of patients being $>50$-years old (range, 15-77). A total of $65(41.7 \%)$ patients had tumors located at the head of the pancreas and $53(34.0 \%)$ patients had tumors $<3 \mathrm{~cm}$ in diameter. In the current series, $65.4 \%$ of patients had stage I or II tumors. A majority (73.1\%) of patients had G1 or G2 diseases, and $15(9.6 \%)$ patients had functional diseases. For all the patients, $\sim 59.0 \%$ of the patients had pNETs with symptoms and the other $41 \%$ were asymptomatic.

The selected cut-off value for CA19-9 as a prognostic predictor of pNETs was $16 \mathrm{U} / \mathrm{ml}$ by the ROC curve (area under ROC curve, 0.68; sensitivity $61.9 \%$; specificity $76.7 \%$; Fig. 1), with $32.7 \%$ of cases having CA19-9 expression levels higher than the cut-off value. A total of $8(5.1 \%)$ cases had a CA19-9 expression level $>100 \mathrm{U} / \mathrm{ml}$ and $22(14.1 \%)$ cases were $>37 \mathrm{U} / \mathrm{ml}$. Univariate analysis was performed in order to evaluate factors associated with overall survival using the 
Table III. Serum CA19-9 expression levels, patient demographics and clinical characteristics.

\begin{tabular}{|c|c|c|c|}
\hline Demographic or clinical characteristic & CA19-9 s16 U/ml & CA19-9 >16 U/ml & P-value \\
\hline Age & & & 0.379 \\
\hline$\leq 50$ years & 49 & 20 & \\
\hline$>50$ years & 56 & 31 & \\
\hline Gender & & & 0.465 \\
\hline Male & 47 & 26 & \\
\hline Female & 58 & 25 & \\
\hline Location & & & 0.931 \\
\hline Head & 44 & 21 & \\
\hline Others & 61 & 30 & \\
\hline Size & & & 0.119 \\
\hline$\leq 3 \mathrm{~cm}$ & 40 & 13 & \\
\hline$>3 \mathrm{~cm}$ & 65 & 38 & \\
\hline TNM stage & & & $0.001^{\mathrm{a}}$ \\
\hline I, II & 78 & 24 & \\
\hline III, IV & 27 & 27 & \\
\hline Grade & & & 0.075 \\
\hline $\mathrm{G} 1, \mathrm{G} 2$ & 82 & 32 & \\
\hline G3 & 8 & 8 & \\
\hline Lymph status ${ }^{\mathrm{b}}$ & & & 0.057 \\
\hline Positive & 12 & 10 & \\
\hline Negative & 45 & 14 & \\
\hline Vessel invasion $^{\mathrm{b}}$ & & & 0.093 \\
\hline Positive & 10 & 8 & \\
\hline Negative & 41 & 15 & \\
\hline Nerve invasion ${ }^{\mathrm{b}}$ & & & 0.429 \\
\hline Positive & 7 & 5 & \\
\hline Negative & 42 & 18 & \\
\hline Functional & & & 0.085 \\
\hline Positive & 13 & 2 & \\
\hline Negative & 92 & 49 & \\
\hline Symptomatic & & & 0.310 \\
\hline Positive & 59 & 33 & \\
\hline Negative & 46 & 18 & \\
\hline
\end{tabular}

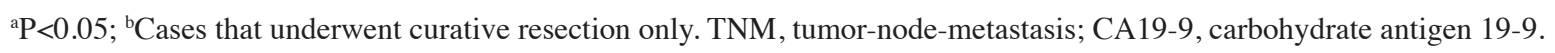

Cox's proportional hazards model. The results demonstrated that TNM stage III or IV $(\mathrm{HR}=5.08 ; \mathrm{P}<0.001)$, CA19-9 $>16 \mathrm{U} / \mathrm{ml}(\mathrm{HR}=2.60 ; \mathrm{P}=0.006)$ and $\mathrm{G} 3$ diseases $(\mathrm{HR}=13.43$; $\mathrm{P}<0.001)$ are adverse prognostic factors for patients' overall survival, whereas age, gender, tumor size and tumor location were not significantly associated with overall survival (Table II; Fig. 2). In multivariate analysis, TNM stage III or IV $(\mathrm{HR}=2.88 ; \mathrm{P}=0.018)$ and $\mathrm{G} 3$ diseases $(\mathrm{HR}=8.79 ; \mathrm{P}<0.001)$ were determined to be adverse prognostic factors for patients' overall survival (Table II).

Parameters associated with baseline NLR levels. A $\chi^{2}$ test was performed in order to compare clinicopathological characteristics between the CA19-9 $\leq 16 \mathrm{U} / \mathrm{ml}$ group and the
CA19-9 >16 U/ml group (Table III). The CA19-9 >16 U/ml group had a significantly higher proportion of patients with TNM stage III/IV $(\mathrm{P}=0.001)$, but not age $(\mathrm{P}=0.379)$, gender $(\mathrm{P}=0.465)$, location $(\mathrm{P}=0.931)$, nerve invasion $(\mathrm{P}=0.429)$, functional $(\mathrm{P}=0.085)$ and symptomatic status $(\mathrm{P}=0.310)$. Although not statistically significant, the CA19-9 >16 U/ml group also demonstrated a trend to have a greater proportion of $\mathrm{G} 3$ tumors $(\mathrm{P}=0.075)$, positive lymph status $(\mathrm{P}=0.057)$, tumor size $(\mathrm{P}=0.119)$ and vessel invasion $(\mathrm{P}=0.093)$.

\section{Discussion}

The present study determined the cut-off value for CA19-9 as a prognostic predictor of pNETs to be $16 \mathrm{U} / \mathrm{ml}$, by ROC curve. 

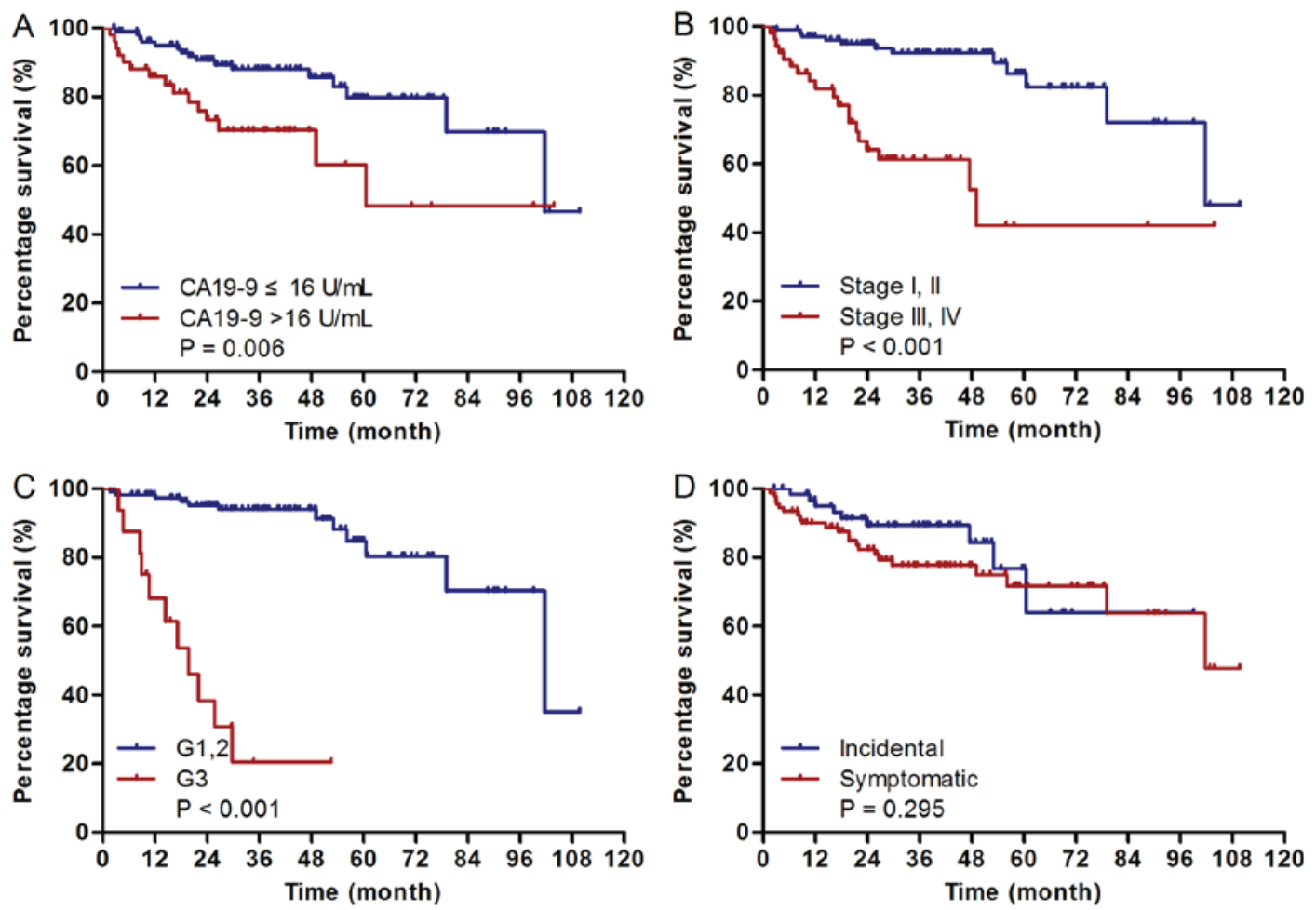

Figure 2. Kaplan-Meier survival curves for patients with pancreatic neuroendocrine tumors according to (A) CA19-9 expression levels ( $\leq 16 \mathrm{U} / \mathrm{ml} \mathrm{vs}$. $>16 \mathrm{U} / \mathrm{ml}$ ), (B) TNM stage (I, II vs. III, IV), (C) grade (G1, G2 vs. G3) and (D) symptoms (incidental vs. symptomatic). CA19-9 >16 U/ml (P=0.006), TNM stage III or IV $(\mathrm{P}<0.001)$ and $\mathrm{G} 3$ diseases $(\mathrm{P}<0.001)$ are adverse prognostic factors for patients' overall survival, whereas symptomatic disease was not significantly associated with overall survival $(\mathrm{P}=0.295)$. CA19-9, carbohydrate antigen 19-9.

Univariate analysis demonstrated that CA19-9 >16 U/ml was an adverse prognostic factor for patients' overall survival. It was also revealed that the CA19-9>16 U/ml group had a statistically higher proportion of TNM stage III/IV, as compared with the CA19-9 $\leq 16 \mathrm{U} / \mathrm{ml}$ group. These findings indicate that CA19-9 may be a prognostic biomarker of pNETs, which may be able to reflect the aggressiveness and severity of the disease.

Chromogranin A $(\mathrm{CgA})$ is currently the most widely used and most characterized biomarker of pNETs, and is detected in the circulation $(16,21-23)$. In contrast to $\mathrm{CgA}$, which is known to be elevated in well- and moderately-differentiated NETs (23), the present study demonstrated that increased CA19-9 expression levels indicated s poor prognosis, G3 disease, advanced stage and aggressive features. Therefore, for cases with abnormal elevation of the CA19-9 expression level, active treatment, including surgical resection and adjuvant treatments, and close follow-up must be strongly recommended and observation alone should not be used. In addition, CA19-9 may supplement $\mathrm{CgA}$ as a prognostic biomarker for pNETs. Furthermore, CgA should be combined with CA19-9 to reflect the tumor volume and severity of pNETs.

Compared with symptomatic non-functional pNETs, incidental pNETs are more frequently $<2 \mathrm{~cm}$ in diameter, stage T1, node negative, grade I and associated with improved prognosis (15). Cheema et al (15) evaluated 143 patients with stage I-III pNETs, and the 5-year progression-free survival rate of incidentally diagnosed tumors was significantly higher compared with symptomatic tumors ( 86.0 vs. $59.0 \%$; $\mathrm{P}=0.007$ ). The present study revealed that $41.0 \%$ of pNETs were incidental pNETs, and these demonstrated no significantly improved survival compared with symptomatic tumors $(\mathrm{HR}=0.67$; $\mathrm{P}=0.295$ ), contrary to the results of a previous study (15).

In the present study cohort, only $8(5.1 \%)$ cases had a CA19-9 expression level $>100 \mathrm{U} / \mathrm{ml}$ and $22(14.1 \%)$ cases were $>37 \mathrm{U} / \mathrm{ml}$, which is a lower frequency compared with that in pancreatic cancer (14). However, the use of CA19-9 as a differentiating diagnostic marker for pancreatic cancer must be utilized with caution, as $>14 \%$ of pNETs were found to have aberrant CA19-9 secretion $(>37 \mathrm{U} / \mathrm{ml})$. The molecular mechanisms underlying the abnormal elevation of CA19-9 expression levels in pNETs are largely undefined. Previous studies have demonstrated that CA19-9 abnormal secretion may be explained by tumor hypoxia and glycosylation $(24,25)$. The observation that CA19-9 was correlated with TNM stage and vessel invasion in pNETs in the present study also indicates that tumor hypoxia and glycosylation may be potential associated mechanisms. Further studies are required in order to confirm this hypothesis.

In the present study, pNETs with a CA19-9 >16 U/ml demonstrated a trend towards having a higher proportion of G3 tumors, as compared with pNETs with a CA19-9 $\leq 16 \mathrm{U} / \mathrm{ml}$ $(\mathrm{P}=0.075)$. G3 pNETs are well known for their aggressiveness and poor response to major treatment strategies, including surgery (26). Considering the differing management strategy between G1, G2 and G3 pNETs in clinical practice, the aberrant elevation of CA19-9 may serve as an indication of G3 pNETs for clinicians (26). However, further studies with larger sample cohort are required.

The novelty of the present study is in that, to the best of our knowledge, it is the first to reveal that CA19-9 is a 
prognostic biomarker of pNETs, which may be able to reflect poor prognosis, advanced stage and aggressive characteristics. This result is in contrast to the results of a previous study that indicated that CA19-9 has limited value in the management of pNETs (27). In addition, CA19-9 may supplement CgA as a biomarker to guide the management of pNETs. However, as the present study was retrospective, the results must be interpreted with caution. Further prospective studies with larges sample sizes are urgently required in order to confirm these findings.

\section{Acknowledgements}

The present study was supported by the National Science Foundation for Distinguished Young Scholars of China (grant no. 81625016), the National Natural Science Foundation of China (grant nos. 81372649, 81172276, 81370065 and 81372653), the Shanghai Municipal Commission of Health and Family Planning Scientific Research (grant no. 20144Y0170) and the Basic Research Projects of the Science and Technology Commission of Shanghai Municipality (grant no. 15JC1401200).

\section{References}

1. Halfdanarson TR, Rabe KG, Rubin J and Petersen GM: Pancreatic neuroendocrine tumors (PNETs): Incidence, prognosis and recent trend toward improved survival. Ann Oncol 19: 1727-1733, 2008.

2. Luo G, Liu Z, Guo M, Jin K, Xiao Z, Liu L, Xu J, Zhang B, Liu C, Huang D, et al: (18)F-FDG PET/CT can be used to detect non-functioning pancreatic neuroendocrine tumors. Int J Oncol 45: 1531-1536, 2014

3. Bilimoria KY, Tomlinson JS, Merkow RP, Stewart AK, Ko CY, Talamonti MS and Bentrem DJ: Clinicopathologic features and treatment trends of pancreatic neuroendocrine tumors: Analysis of 9,821 patients. J Gastrointest Surg 11: 1460-1469, 2007.

4. Du S, Wang Z, Sang X, Lu X, Zheng Y, Xu H, Xu Y, Chi T, Zhao H, Wang W, et al: Surgical resection improves the outcome of the patients with neuroendocrine tumor liver metastases: Large data from Asia. Medicine (Baltimore) 9: e388, 2015.

5. Frilling A, Akerström G, Falconi M, Pavel M, Ramos J, Kidd M and Modlin IM: Neuroendocrine tumor disease: An evolving landscape. Endocr Relat Cancer 19: R163-R185, 2012.

6. Falconi M, Zerbi A, Crippa S, Balzano G, Boninsegna L, Capitanio V, Bassi C, Di Carlo V and Pederzoli P: Parenchyma-preserving resections for small nonfunctioning pancreatic endocrine tumors. Ann Surg Oncol 17: 1621-1627, 2010.

7. Valle JW, Eatock M, Clueit B, Gabriel Z, Ferdinand R and Mitchell S: A systematic review of non-surgical treatments for pancreatic neuroendocrine tumours. Cancer Treat Rev 40: 376-389, 2014.

8. Yang M, Zeng L, Zhang Y, Su AP, Yue PJ and Tian BL: Surgical treatment and clinical outcome of nonfunctional pancreatic neuroendocrine tumors: A 14-year experience from one single center. Medicine (Baltimore) 93: e94, 2014.

9. Kuo EJ and Salem RR: Population-level analysis of pancreatic neuroendocrine tumors $2 \mathrm{~cm}$ or less in size. Ann Surg Oncol 20: 2815-2821, 2013.

10. Lee LC, Grant CS, Salomao DR, Fletcher JG, Takahashi N, Fidler JL, Levy MJ and Huebner M: Small, nonfunctioning, asymptomatic pancreatic neuroendocrine tumors (PNETs): Role for nonoperative management. Surgery 152: 965-974, 2012.
11. Liu B, Tang LH, Liu Z, Mei M, Yu R, Dhall D, Qiao XW, Zhang TP, Zhao YP, Liu TH, et al: $\alpha$-Internexin: A novel biomarker for pancreatic neuroendocrine tumor aggressiveness. J Clin Endocrinol Metab 99: E786-E795, 2014.

12. Koprowski H, Steplewski Z, Mitchell K, Herlyn M, Herlyn D and Fuhrer P: Colorectal carcinoma antigens detected by hybridoma antibodies. Somatic Cell Genet 5: 957-971, 1979.

13. Luo G, Xiao Z, Long J, Liu Z, Liu L, Liu C, Xu J, Ni Q and Yu X: CA125 is superior to CA19-9 in predicting the resectability of pancreatic cancer. J Gastrointest Surg 17: 2092-2098, 2013.

14. Goonetilleke KS and Siriwardena AK: Systematic review of carbohydrate antigen (CA 19-9) as a biochemical marker in the diagnosis of pancreatic cancer. Eur J Surg Oncol 33: 266-270, 2007.

15. Cheema A, Weber J and Strosberg JR: Incidental detection of pancreatic neuroendocrine tumors: An analysis of incidence and outcomes. Ann Surg Oncol 19: 2932-2936, 2012.

16. Hijioka M, Ito T, Igarashi H, Fujimori N, Lee L, Nakamura T, Jensen RT and Takayanagi R: Serum chromogranin A is a useful marker for Japanese patients with pancreatic neuroendocrine tumors. Cancer Sci 105: 1464-1471, 2014.

17. Elisei R, Lorusso L, Romei C, Bottici V, Mazzeo S, Giani C, Fiore E, Torregrossa L, Insilla AC, Basolo F, et al: Medullary thyroid cancer secreting carbohydrate antigen 19-9 (Ca 19-9): A fatal case report. J Clin Endocrinol Metab 98: 3550-3554, 2012.

18. Elisei R, Lorusso L, Piaggi P, Torregrossa L, Pellegrini G, Molinaro E, Agate L, Bottici V, Pani F, Cacciato Insilla A, et al: Elevated serum levels of carbohydrate antigen 19.9 (Ca 19.9) is a prognostic factor of death in patients with advanced medullary thyroid cancer. Eur J Endocrinol 173: 297-304, 2015.

19. Luo G, Javed A, Strosberg JR, Jin K, Zhang Y, Liu C, Xu J, Soares K, Weiss MJ, Zheng L, et al: Modified staging classification for pancreatic neuroendocrine tumors on the basis of the American Joint Committee on Cancer and European Neuroendocrine Tumor Society Systems. J Clin Oncol 35: 274-280, 2017

20. Rindi G, Arnold R, Bosman FT, Capella C, Klimstra D and Klöppel G: Nomenclature and classification of neuroendocrine neoplasms of the digestive system. In: Bosman TF, Carneiro F, Hruban RH, Theise ND (eds). WHO classification of tumours of the digestive system. Lyon, France, International Agency for Research on Cancer (IARC), 2010.

21. Campana D, Nori F, Piscitelli L, Morselli-Labate AM, Pezzilli R, Corinaldesi R and Tomassetti P: Chromogranin A: Is it a useful marker of neuroendocrine tumors? J Clin Oncol 25: 1967-1973, 2007.

22. Zatelli MC, Torta M, Leon A, Ambrosio MR, Gion M, Tomassetti P, De Braud F, Delle Fave G, Dogliotti L and degli Uberti EC; Italian CromaNet Working Group: Chromogranin A as a marker of neuroendocrine neoplasia: An Italian Multicenter Study. Endocr Relat Cancer 14: 473-482, 2007.

23. Modlin IM, Gustafsson BI, Moss SF, Pavel M, Tsolakis AV and Kidd M: Chromogranin A-biological function and clinical utility in neuro endocrine tumor disease. Ann Surg Oncol 17: 2427-2443, 2010.

24. Kannagi R: Carbohydrate antigen sialyl Lewis a-its pathophysiological significance and induction mechanism in cancer progression. Chang Gung Med J 30: 189-209, 2007.

25. Kannagi R, Sakuma K, Miyazaki K, Lim KT, Yusa A, Yin J and Izawa M: Altered expression of glycan genes in cancers induced by epigenetic silencing and tumor hypoxia: Clues in the ongoing search for new tumor markers. Cancer Sci 101: 586-593, 2010.

26. Jin K, Xu J, Chen J, Chen M, Chen R, Chen Y, Chen Z, Cheng B, Chi Y, Feng ST, et al: Surgical management for non-functional pancreatic neuroendocrine neoplasms with synchronous liver metastasis: A consensus from the Chinese Study Group for Neuroendocrine Tumors (CSNET). Int J Oncol 49: 1991-2000, 2016

27. Li J, Luo G, Fu D, Jin C, Hao S, Yang F, Wang X, Yao L and Ni Q: Preoperative diagnosis of nonfunctioning pancreatic neuroendocrine tumors. Med Oncol 28: 1027-1031, 2011. 